\title{
Full neuroendoscopic contralateral longitudinal fissure approach to resection of parafalcine meningioma via falx cerebri - Retractorless surgical technique: a case report
}

Changchen Hu ( $\nabla$ huchangchen666@gmail.com )

Shanxi Medical University

Jiangtao Liu

Shanxi Medical University

Hongming Ji

Shanxi Medical University

Gangli Zhang

Shanxi Medical University

Shengli Chen

Shanxi Medical University

Shiyuan Zhang

Shanxi Medical University

Kaixuan Wang

Shanxi Medical University

\section{Case report}

Keywords: parafalcine meningioma, full endoscopic surgery, contralateral longitudinal fissure approach, retractorless surgical technique

Posted Date: November 17th, 2020

DOI: https://doi.org/10.21203/rs.3.rs-104877/v1

License: (c) (1) This work is licensed under a Creative Commons Attribution 4.0 International License. Read Full License 


\section{Abstract}

Background: Parafalcine meningioma often invades into contralateral hemisphere, and sometimes it can develop to both sides. An ipsilateral craniotomy is generally performed for the surgical resection of parafalcine meningioma.

Case report: Here, we report that a 50-year-old female with a right anterior cerebral parafalcine meningioma. The patient was in supine position and the head was rotated 20 degrees to the opposite side. A neuronavigation was employed to perform a precise surface localization of parafalcine meningioma. Under neuroendoscopy, a contralateral craniotomy and a longitudinal fissure approach were followed by an incision of dura to expose arachnoid, then arachnoid was separated until falx cerebri along its surface. Neuronavigation was employed again to precisely locate the projection of meningioma in the contralateral falx cerebri. According to the position, firstly removed the invaded falx cerebri, blocked the blood supply of the meningioma, then completely resected the meningioma without retraction of normal brain tissue.

Conclusion: We conclude that full neuroendoscopic contralateral longitudinal fissure approach to complete resect parafalcine meningioma via falx cerebri using retractorless surgical technique can reduce the retraction of normal brain tissue to enhance protection of brain function, while maximizing the total resection rate of parafalcine meningioma.

\section{Introduction}

Parafalcine meningioma refers to a common type of meningioma located in the longitudinal fissure and is connected to falx cerebri. Except its large growing space and plaque-like growth, parafalcine meningioma is barely involved in the superior sagittal sinus. It often invades into contralateral hemisphere, and sometimes it can develop to both sides [1]. An ipsilateral craniotomy is generally performed for the surgical resection of parafalcine meningioma. Along the longitudinal fissure, the cortical area without bridge veins is selected, and the cerebral hemisphere is retracted from the falx cerebri by a retractor under the microscope. The tumor is gradually explored until it is reached. The tumor is first devascularized, then separated and finally dissected from the falx cerebri [2]. However, after years of clinical practice, it is found that an improper use or an over-reliance of brain automatic retractor to retract the ipsilateral cerebral hemisphere may lead to ipsilateral postoperative cerebral edema, and further lead to the contralateral limb paralysis, sensory disturbance, and visual field deficits [3]. With the rapid development of neuroendoscopy in recent years, we have explored the application of full endoscopic contralateral longitudinal fissure approach via the falx cerebri to remove parafalcine meningiomas without retractor.

\section{Presentation And Examination}


Female, 50 years old, married. Han Chinese, farmer. The patient was admitted to hospital in August and her chief complaint was numbness of the left lower extremity. The patient reported that there was no obvious cause for the numbness and stiff in the left upper extremity before August. Afterwards, she started to feel numbness in her left lower extremity, but no syndromes of headache, dizziness, or facial palsy. She was admitted in a local hospital. CT and MRI scan showed a tumor on the right frontoparietal with edema (Fig. 1). Physical examination: Her left limbs had milder sensations of temperature, tactile and pain than the right side, muscle strength was grade IV, and Babinski sign was positive on the left. Diagnosis of administration: parafalcine meningioma on the right frontoparietal.

\section{Surgical Treatment}

The patient underwent general anesthesia. A full endoscopic left longitudinal fissure approach was employed to resect right parafalcine meningioma. After the head frame was fixed, the head was rotated $30^{\circ}$ toward front, which exposed the operation area on the frontoparietal. At the same time, the head was rotated $15-20^{\circ}$ toward to the left side, allowing the gravity retracted the brain tissue in front lobe towards the left side. This method can expose more natural cavity of the longitudinal fissure and allow endoscopy entering the cavity. Upon the retractorless technique, the left falx cerebri was exposed to perform the devascularization of right parafalcine meningioma. Tumor debulking was operated first to reduce the tumor size, then tumor was separated from the arachnoid of right frontoparietal and finally resected. After the operation, the patient had a transient right limbs numbness with weak muscle strength (Grade IV). One week after the operation, the muscle strength of bilateral limbs was completely recovered (Grade V). Postoperative CT and MRI confirmed a complete resection of the tumor (Fig. 2.).

\section{Discussion}

Parafalcine meningioma is difficult to expose due to its deep location in the cerebral longitudinal fissure. Generally, the ipsilateral craniotomy is performed. Under the microscope, the cerebral hemisphere is retracted from the falx cerebri by a brain retractor. The tumor base is first devascularized and then dissected from the surrounding cerebral structures. After tumor is completely removed, the base on the falx cerebri is electrocauterized [4,5]. However, this approach has its limitations: 1 . The meningioma is deeply buried in the ipsilateral cerebral longitudinal fissure. It requires a use of brain retractor to retract hemisphere from the falx cerebri. This operation may lead to postoperative intracerebral hematoma and injury, retractor-induced edema, or ischemic injury due to retractor-induced decrease in cerebral blood perfusion resulting from a direct compression to the cortex and subcortical tissues [6]. 2. The tumor has to be devascularized at its base. Debulking and devascularization has to be done at the same time due to the block from tumor itself, which easily lead to the excessive blood loss and is not conducive to postoperative recovery. In some cases, sometimes, it is even required to remove the brain tissues in frontal or occipital lobes to expose tumor [7].

Soumya Mukherjee reported the resection of a parafalcine meningioma from the contralateral longitudinal fissure approach under the microscope. It can avoid the retraction of cortex to expose tumor 
and reduce the retraction-induced brain injury and edema. At the same time, it can expose the falx first to facilitate the devascularization of meningioma [8]. However, it is still required to retract the brain tissue using retractor to expose the lateral fissure for surgery, which cannot achieve a complete retractorless. Thus, it may still lead to retractor-induced edema, or a direct compression to the cortex and subcortical tissues.

The rapid development of neuroendoscopy in recent years has provided us a basis for exploring the resection of parafalcine meningioma without a retractor. The surgical position is rotated $15-20^{\circ}$ to the opposite side, and the contralateral cerebral lobe is collapsed laterally by gravity, so that the natural cavity of the longitudinal fissure is exposed as wide as possible. The endoscope can be directly inserted into the longitudinal fissure and reach the lower boundary of the falx cerebri- the lower sagittal sinus without any brain retractor to facilitate the exposure of the longitudinal fissure. When the operation begins to excise the falx cerebri, the endoscopic close observation and multi-angle observation are used to assist the tumor devascularization from any part of contralateral falx cerebri without any retractor. The falx cerebri can be more widely exposed and excised to achieve SIMPSON Grade I resection and reduce recurrence. When separate the arachnoid between the tumor and the surrounding normal brain tissues, the ipsilateral approach is vertical to the tumor boundary, while the contralateral approach enters at an oblique angle that reduces the retraction of brain tissues. Combining endoscopic close- and multi-angle observations, the contralateral approach can completely separate the arachnoid between tumor and surrounding normal brain tissues and reduce the retraction-induced brain edema and injury (Fig. 3). In addition, in this case, the ipsilateral brain surface has a thick drainage vein, which also increases the difficulty of ipsilateral approach. Once the drainage vein of the functional area is damaged, it is easy to cause severe postoperative dysfunction or even unrecoverable dysfunction. Moreover, in this case, arachnoid cysts were observed on the contralateral side and the cerebral cortex was significantly lower than the contralateral side, which also created conditions for the contralateral approach. In our another case, we observed the contralateral arachnoid cyst and the significantly lower cortex. Whether the contralateral arachnoid cysts are accidental events or patterns remains unknown and it needs further studies.

\section{Conclusion}

This case of complete resection of parafalcine meningiomas was completely under neuroendoscopy, and used the retractorless technique and a contralateral longitudinal fissure approach via falx cerebri. There was no obvious brain injury or intracerebral hematoma, which reduced postoperative complications caused by retraction. The development of modern neuroendoscopy technology, using its slimness, closed observation and multi-angle observation, can maximally enhance the advantages of retractorless surgical technique, and provide a minimally invasive surgical method for surgical resection of parafalcine meningioma.

\section{Abbreviations}


MRI

Magnetic resonance imaging; CT:Computed tomography

\section{Declarations}

\section{Acknowledgment}

The authors respectfully thank the patient and her family for their trust and agreement on the publication of this case. We are grateful to all the medical staff involved in the surgical treatment of the patient, including those in the department of blood transfusion and the operating room.

\section{Funding}

This research was funded by the National Natural Science Foundation of China (30901774), National Science Foundation of Shanxi Province (2014011038-2) and Shanxi Provincial Key Research and Development Project (201803D421055).

\section{Availability of data and materials}

Data sharing is not applicable to this article as no datasets were generated or analyzed during the current study.

\section{Authors' contributions}

$\mathrm{JL}$ and $\mathrm{CH}$ conceived and designed the work and drafted the manuscript. $\mathrm{HJ}, \mathrm{GZ}, \mathrm{SC}$ and $\mathrm{SZ}$ conceived and designed the work and revised the manuscript. KW acquired the data and participated the operation. $\mathrm{CH}$ performed the operation and approved the final version. All authors read and approved the final manuscript.

\section{Ethics approval and consent to participate}

This article has been approved by the ethics committee of Shanxi Provincial People's Hospital, Shanxi Medical University.

\section{Consent for publication}

The patient had signed the agreement and agreed with the publication of this paper.

\section{Competing interests}

The authors declare that they have no competing interests.

\section{References}


1. Das KK, Gosal JS, Sharma P, Mehrotra A, Bhaisora K, Sardhara J, Srivastava A, Jaiswal AK, Kumar R, Behari Ss. Falcine Meningiomas: Analysis of the Impact of Radiologic Tumor Extensions and Proposal of a Modified Preoperative Radiologic Classification Scheme. World Neurosurg. 2017;104:248-258.

2. Murrone D, De Paulis D, di Norcia V, Di Vitantonio H, Galzio RJ. Surgical management of falcine meningiomas: Experience of 95 patients. J Clin Neurosci. 2017;37:25-30.

3. Sughrue ME, Rutkowski MJ, Shangari G, Parsa AT, Berger MS, McDermott MW. Results with judicious modern neurosurgical management of parasagittal and falcine meningiomas. Clinical article. $J$ Neurosurg. 2011;114(3):731-7.

4. Ransohoff J. Removal of convexity, parasagittal, and falcine meningiomas. Neurosurg Clin N Am. 1994;5(2): 293-7.

5. Nowak A, Dziedzic T, Czernicki T, Kunert P, Marchel A. Surgical treatment of parasagittal and falcine meningiomas invading the superior sagittal sinus. Neurol Neurochir Pol. 2014;48(3):174-80.

6. Wang X, Wang MY, Qian K, Chen L, Zhang FC. Classification and protection of peritumoral draining veins of parasagittal and falcine meningiomas. World Neurosurg. 2018;14. pii: S18788750(18)31252-X.

7. Bederson JB, Eisenberg MB. Resection and replacement of the superior sagittal sinus for treatment of a parasagittal meningioma: technical case report. 1995;37(5):1015-8.

8. Mukherjee S, Minhas PS. Contralateral approach to resection of a parafalcine meningioma-a technical note. Br J Neurosurg. 2014;28(5):699-700.

\section{Figures}



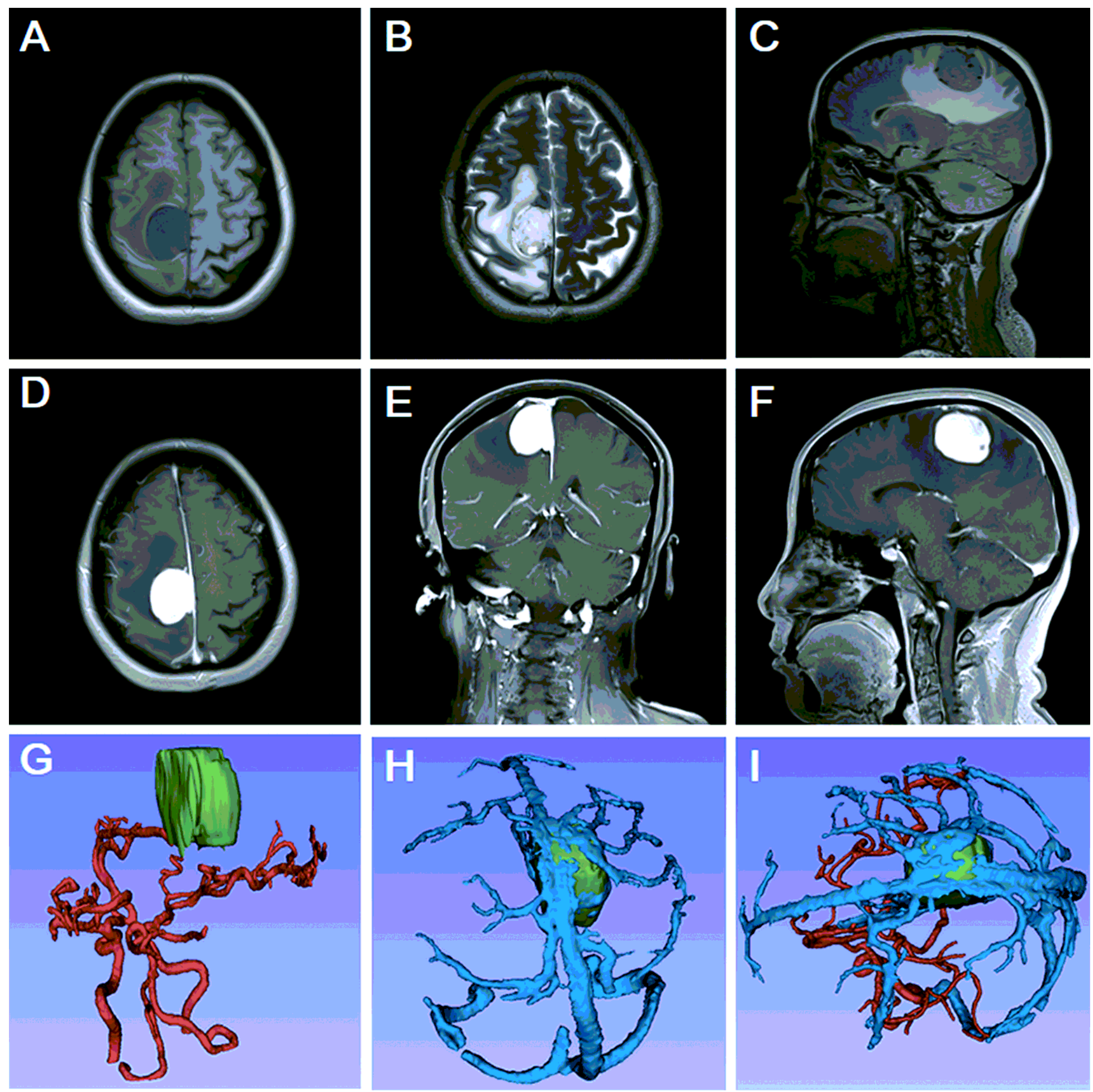

Figure 1

Preoperative images of parafalcine meningioma. A-C: Preoperative axial and sagittal scans; D-F: Preoperative axial, coronal and sagittal enhanced scans. The coronal scan (E) revealed an arachnoid cyst in the contralateral (left) side near the midline, which created a condition for reducing hemisphere retraction; G-I: Preoperative venous reconstruction image showing a thick drainage vein on the surface of ipsilateral hemisphere, which blocked the ipsilateral approach for the resection of the parafalcine 
meningioma. However, no large vein was observed blocking the approach at contralateral hemisphere, which created a condition for the contralateral approach.
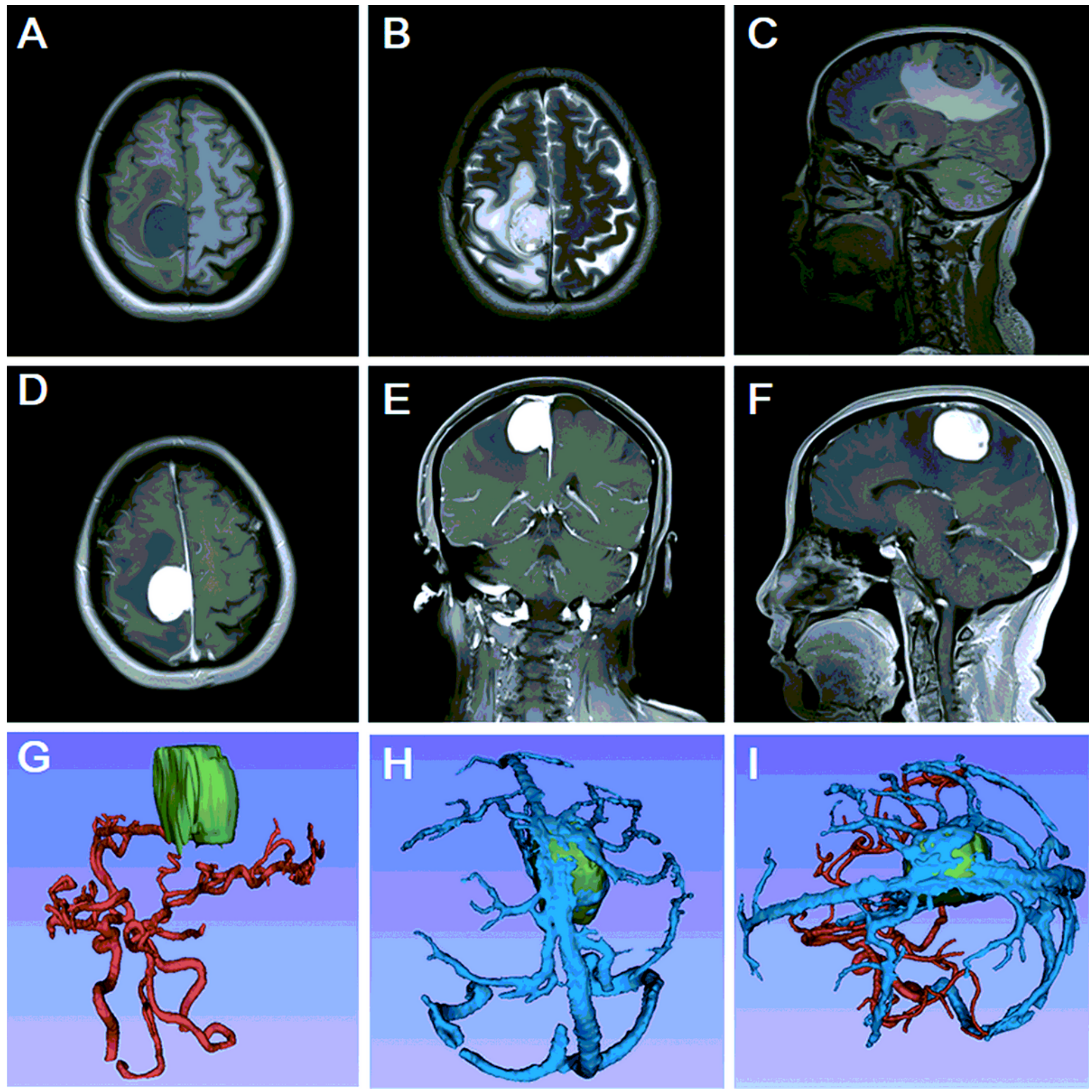

\section{Figure 1}

Preoperative images of parafalcine meningioma. A-C: Preoperative axial and sagittal scans; D-F: Preoperative axial, coronal and sagittal enhanced scans. The coronal scan (E) revealed an arachnoid cyst in the contralateral (left) side near the midline, which created a condition for reducing hemisphere retraction; G-I: Preoperative venous reconstruction image showing a thick drainage vein on the surface of 
ipsilateral hemisphere, which blocked the ipsilateral approach for the resection of the parafalcine meningioma. However, no large vein was observed blocking the approach at contralateral hemisphere, which created a condition for the contralateral approach.
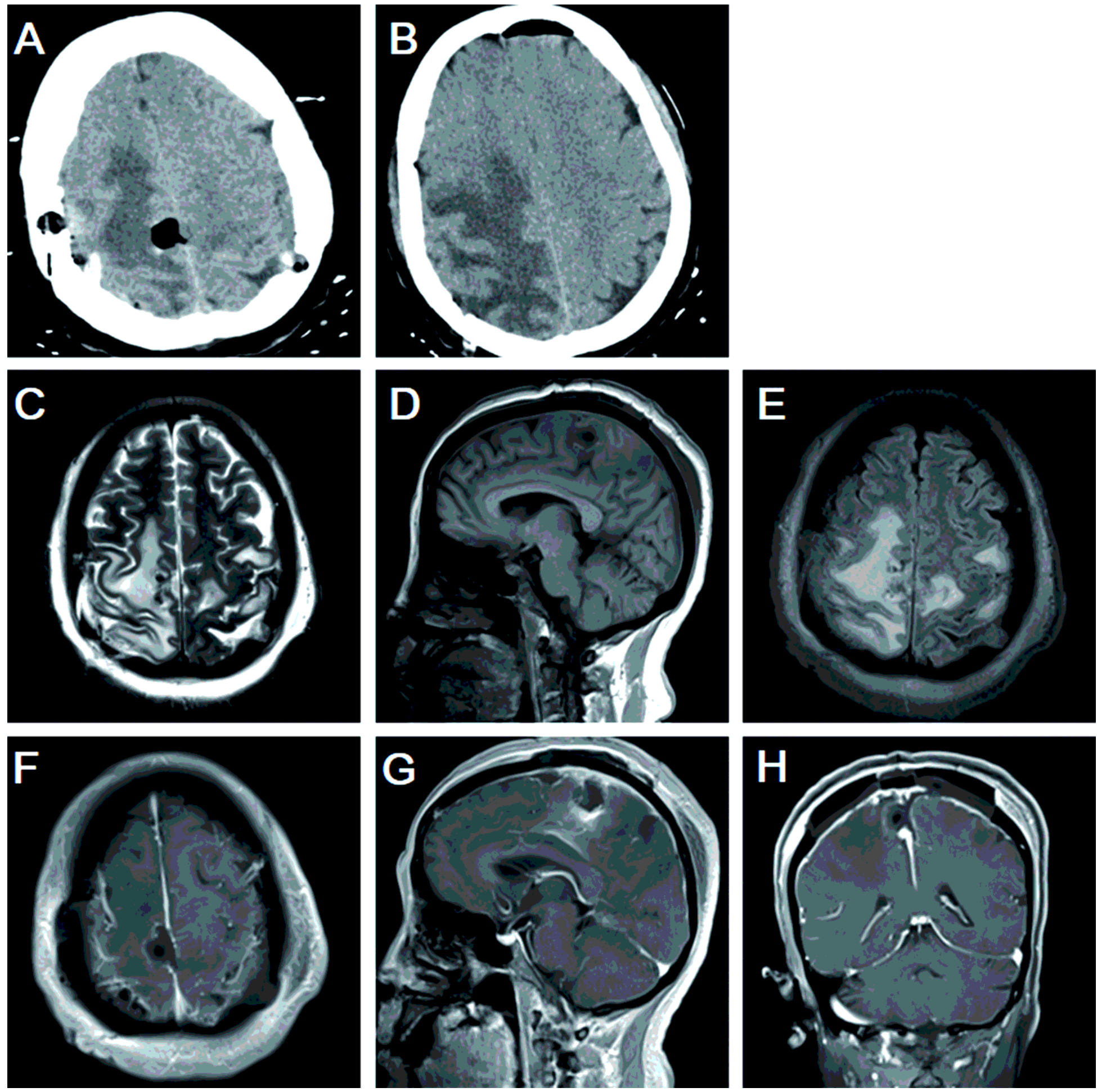

Figure 2

Postoperative images of parafalcine meningioma. A-B: Postoperative CT scans; C-E: Postoperative axial and sagittal scans; F-H: Postoperative axial, coronal and sagittal enhanced scans. Postoperative CT and 
MRI confirmed a complete resection of the tumor. There was no significant increase in cerebral edema compared with preoperative MRI.
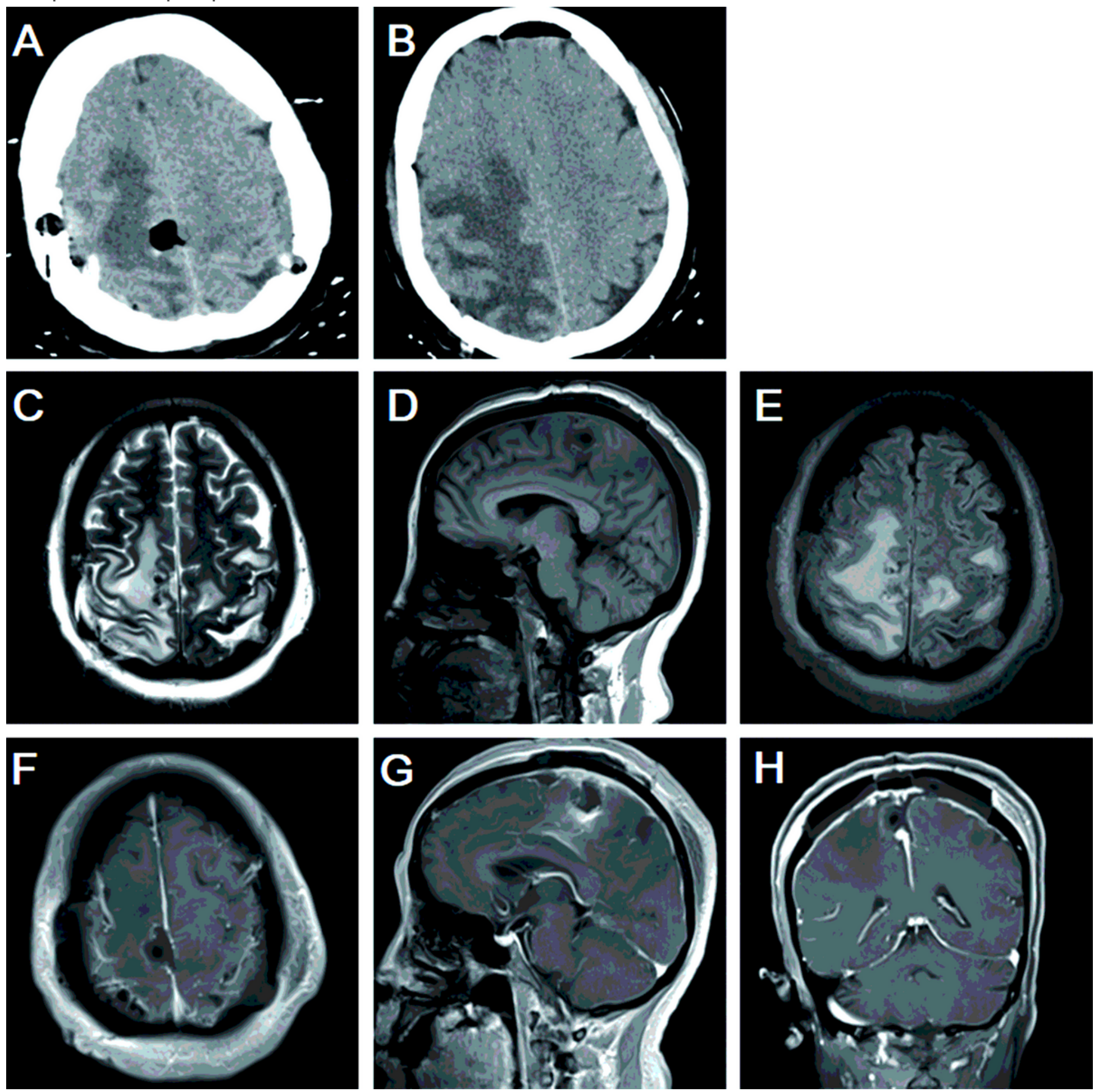

Figure 2

Postoperative images of parafalcine meningioma. A-B: Postoperative CT scans; C-E: Postoperative axial and sagittal scans; F-H: Postoperative axial, coronal and sagittal enhanced scans. Postoperative CT and MRI confirmed a complete resection of the tumor. There was no significant increase in cerebral edema compared with preoperative MRI. 


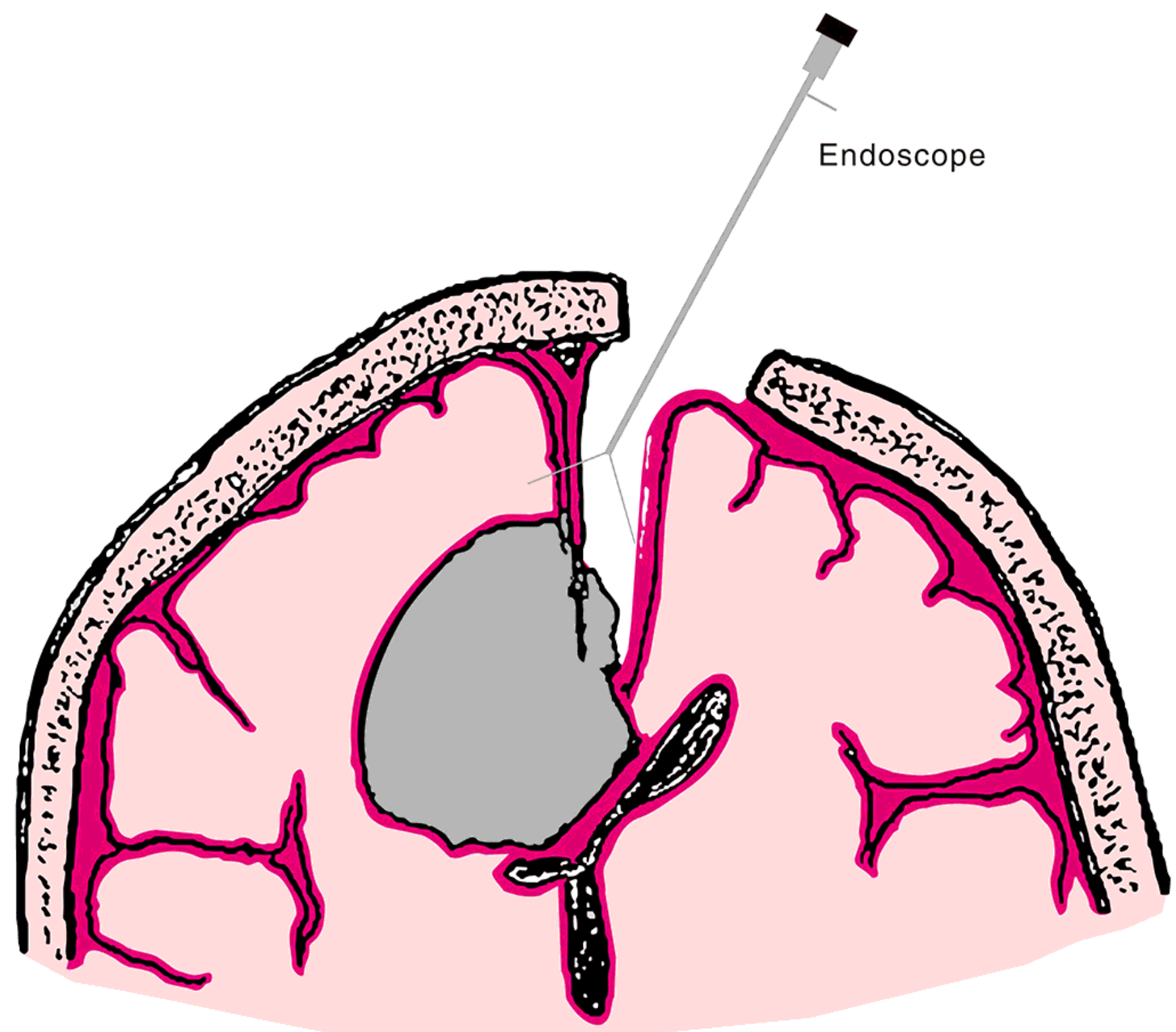

\section{Figure 3}

Artist's illustration of full neuroendoscopic contralateral longitudinal fissure approach to resection of parafalcine meningioma via falx cerebri - Retractorless surgical technique. 


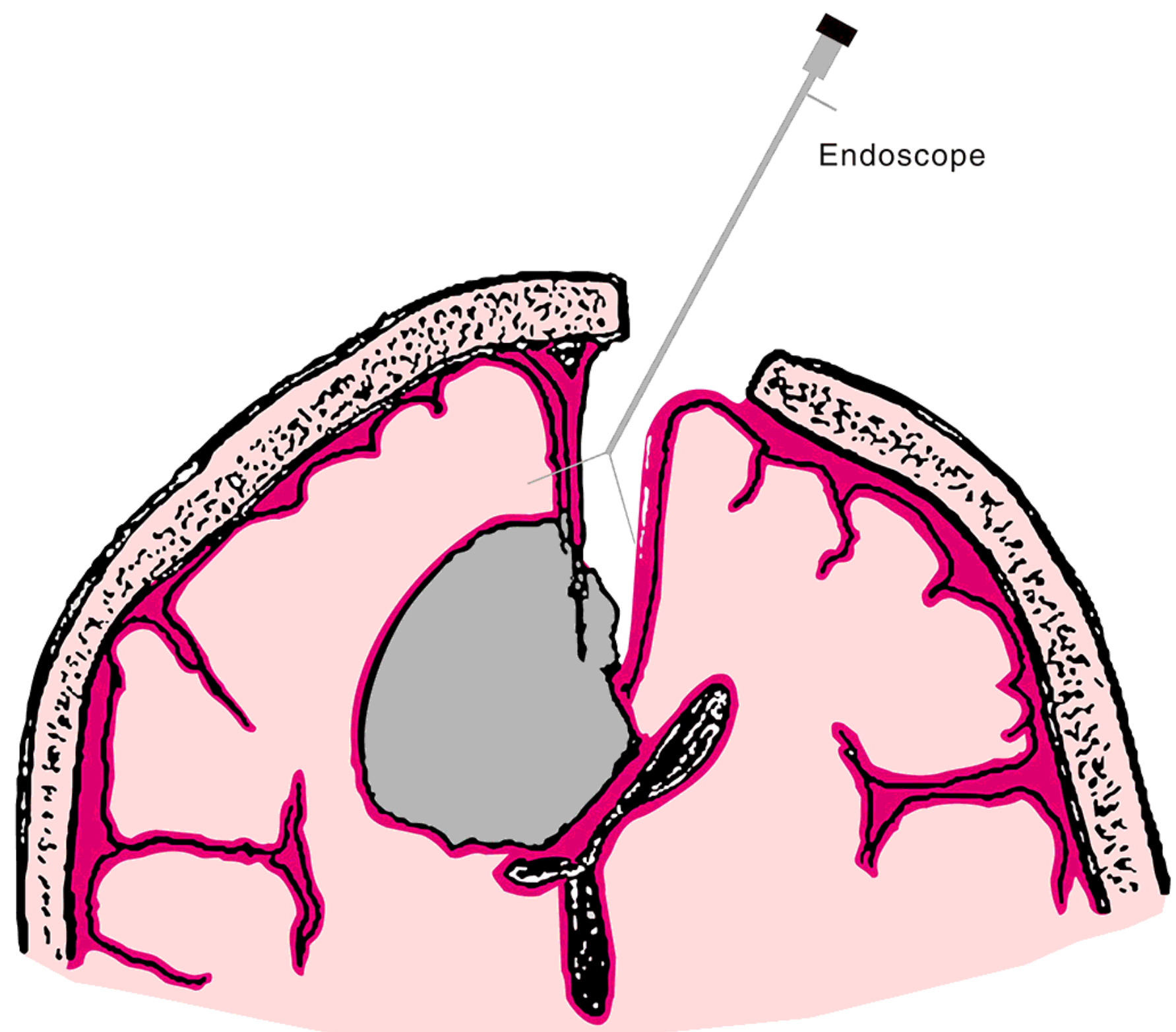

\section{Figure 3}

Artist's illustration of full neuroendoscopic contralateral longitudinal fissure approach to resection of parafalcine meningioma via falx cerebri - Retractorless surgical technique. 